\title{
TWO-COLOR CCD PHOTOMETRY OF THE INTERMEDIATE POLAR 1RXS J180340.0+401214
}

\author{
Ivan L. Andronov ${ }^{1}$, YongGi Kim²,3,4, Joh-Na Yoon ${ }^{2}$, Vitali V.Breus ${ }^{1}$, Tammy A. Smecker-Hane ${ }^{4}$, \\ Lidia L. Chinarova ${ }^{5}$, and WONYONG Han ${ }^{6}$ \\ ${ }^{1}$ Department of High and Applied Mathematics, Odessa National Maritime University, \\ Mechnikov str., 34, Odessa, 65029, Ukraine \\ E-mail :tt_ari@ukr.net,il-a@mail.ru,uavso@pochta.ru \\ ${ }^{2}$ University Observatory, Chungbuk National University, Cheongju 361-763, Korea \\ E-mail : ykkim153@chungbuk.ac.kr,antalece@chungbuk.ac.kr,bvv_2004@ua.fm \\ ${ }^{3}$ Institute for Basic Science Research, Chungbuk National University, Cheongju 361-763, Korea \\ ${ }^{4}$ Department of Physics and Astronomy, 4129 Frederick Reines Hall, University of California, Irvine, \\ CA 92697-4575, USA \\ E-mail : tsmecker@uci.edu \\ ${ }^{5}$ Astronomical Observatory, Odessa National University, Marazlievskaya Str., 1-V, Odessa, 65014, Ukraine \\ E-mail : chinarova@pochta.ru \\ ${ }^{6}$ Korea Astronomy Observatory and Space Science Institute, Daejeon 305-348, Korea \\ E-mail : whan@kasi.re.kr \\ (Received March 23, 2011; Revised April 29, 2011; Accepted May 02, 2011)
}

\begin{abstract}
We present results of two-color VR photometry of the intermediate polar RXS J1803. The data were aquired using the Korean 1-m telescope located at Mt. Lemmon, USA. Different "high" and "low" luminosity states, similar to other intermediate polars, were discovered. No statistically significant variability of the color index with varying luminosity was detected. The orbital variability was found to be not statistically significant. Spin maxima timings were determined, as well as the photometric ephemeris for the time interval of our observations. The spin period variations, caused by interaction of the accretion structure with the rotating magnetic white dwarf, were also detected. These variations are of complicated character, and their study requires further observations. We determine the color transformation coefficients for our photometric systems, and improve on the secondary photometric standards.
\end{abstract}

Key words : stars: individual — 1RXS J180340.0+401214, USNO-A2.0 1275-09738647 — stars: binary — stars: variable — stars: cataclysmic

\section{INTRODUCTION}

Cataclysmic variable stars are excellent natural laboratories to study various astrophysical processes (cf., Warner 1995; Hellier 2001). The total number of main characteristic processes is 23 for a whole class of cataclysmic variables (CVs), slightly smaller in the corresponding sub-classes (see Andronov 2007, 2008 for recent reviews). The basic model of a cataclysmic variable is a close binary system with a red dwarf, which fills its Roche lobe and loses its atmosphere through the vicinities of the inner Lagrangian point. The primary star in the system is a compact white dwarf, which is typically more massive than the secondary (red dwarf). These systems are called close binary systems for a reason that an orbital separation is of order of a solar radius. Thus their periods typically range from 82 to 120 minutes (short-period CVs), from 180

Corresponding Author: Y. Kim to 300 minutes (long-period CVs) and even to 2 days ( $\mathrm{T}$ $\mathrm{CrB})$. According to state-of-the-art theoretical models, a white dwarf accretes matter in two forms: either accretion belt (so-called "non-magnetic" CVs) or accretion column ("magnetic" CVs, or MCVs). Recent studies pointed out the importance of the magnetic field, at least, at close vicinities of the white dwarf (cf., Norton et al. 1999). However, the "zoo" of CVs is typically classified into several main classes: Novae (N), Nova-Like (NL), dwarf Novae (UG, i.e., U Gemtype stars), intermediate polars (IP or DQ, i.e., DQ Her-type stars) and classical polars (AM, i.e., AM Hertype stars). Some stars show characteristics, which are typical for few classes, so there are groups of recurrent novae (RN), asynchronous polars and outbursting intermediate polars (= magnetic dwarf novae). It is usually assumed that all these systems undergo classical Nova outbursts with a recurrence time of $10^{3}-10^{5}$ years. Between the outbursts, different classes of cataclysmic variables show different characteristics. 
From a theoretical point of view, the behavior of the system (and thus its classification) depends on the parameters of the system (i.e. masses and radii of component stars, orbital separation, eccentricity and inclination), accretion rate (amount of mass accreted per unit time), the magnetic field, i.e. more concretely, on the radius of magnetosphere $R_{A}$ (Alfvén Radius), co-rotation radius $R_{\omega}$, radius of the white dwarf $R_{w d}$ and distance from the center of the white dwarf to the inner Lagrangian point $R_{L}$. For "non-magnetic" stars, $R_{w d} \sim R_{A} \sim R_{\omega} \ll R_{L}$, and the accretion disk is being formed - relatively stable, as in the nova-like, or cyclically, as in dwarf novae. For "classical" polars, $R_{w d} \ll R_{A} \sim R_{\omega} \sim R_{L}$.

Between these two extreme classes, there is a very interesting class of magnetic cataclysmic variables, called "intermediate polars". They often show two main types of variability: the "orbital" one caused by rotation of the binary system with a period of (typically) 3-7 hours and the "spin" one (from a few to dozens of minutes) caused by intrisic faster rotation of the magnetic white dwarf with plasma falling via accretion columns. Sometimes "spin-orbital" beat periods are observed (see the classical review by Patterson 1994 for more details). Because the accreting matter transfers mass and angular momentum to the white dwarf, it undergoes period variations, which may be (and should be) studied photometrically. Some systems show period variations, which are caused by varying mass transfer of the system and by varying orientation of the magnetic axis and/or rotational axis (precession) of the white dwarf (e.g., Andronov 2007, 2008)

To study dependence of the characteristics of cataclysmic variables on time and luminosity state, an extensive monitoring of the group of key objects of different classes is needed, initiated in 1978 by Prof. V. P. Tsessevich (1907-1983) and is carried out since then as an international "Inter-Longitude Astronomy" (ILA) project (see e.g., Andronov et al. 2003, 2010 for a sample review of the the highlights). The total number of publications on the project listed in the ADS is currently 310. Since 2003, such an extensive monitoring of the cataclysmic variables (as a part of this general "ILA" project) was initiated in Korea by Prof. Kim Yonggi mainly by using the $1.8 \mathrm{~m}$ telescope of the Bohyunsan optical astronomical observatory and the 1$\mathrm{m}$ Korean telescope at the Mt. Lemmon Observatory (USA). Results of this campaign on selected stars were published by Kim et al. (2004, 2005ab, 2009) and by Andronov et al. (2005abc, 2008ab); some other stars are being further monitored.

The object 1RXS J180340.0+401214 (hereafter RXJ 1803 ) is one of the few newly discovered intermediate polars, which were included in our monitoring list. Although the preliminary period, co-ordinates and the main characteristics were published in the corresponding discovery paper by G'ansicke et al. (2005), we focus on these objects assuming the following main goals: 1) study of the dependence of the characteristics of variability on either time, or luminosity, as these objects, like other cataclysmic variables, exhibit high and low luminosity state and transitions between them: 2) derive the two-color VR photometry, which, contrary to the majority of one-color (or even "clear light" = "unfiltered") CCD observations, allows one to estimate the color index information for different components of variability (i.e., "spin" variability due to rotation of the magnetic white with one or two visible accretion columns; "orbital" variability due to the rotation of the system as a binary (which is only present in the classical eclipsing binaries); "luminosity" changes): 3 ) determine the precise ephemeris and the extrema timings, which will be useful for future studies of the spin period variations (i.e., rotational evolution of the magnetic white dwarf).

Since its discovery, RXJ 1803 was included to our list for monitoring. Its coordinates are R. A. $=18^{\mathrm{h}} 03^{\mathrm{m}}$ $39.67^{\mathrm{s}}$, Dec. $=+40^{\circ} 12^{\mathrm{m}} 20.6^{\mathrm{s}}(2000.0)$ (Gänsicke et al. 2005), other designation is USNO-A2.0 1275-09738647 (Ritter and Kolb 2003-2010). Only two more papers were published on this object, except catalogues. This shows that the system is still badly studied. Teichgraeber et al. (2007) confirmed the periods reported in the discovery paper, although the apparently slight difference in periods causes errors in cycle counts. Anzolin et al. (2008) detected X-ray variability at the spin period of $1520.5 \mathrm{~s}$ and also a "spin-orbital beat" variability at 1697s.

According to the "General Catalogue of Variable Stars" (Samus' et al. 2009-2011), SIMBAD (2011) and VSX (Watson 2006-2011), the object has no official name yet, as a variable star. It is located in the Hercules constellation, as the prototypes of X-ray polars (HZ Her), classical polars (AM Her) and intermediate polars (DQ Her) - see the catalogue of Ritter and Kolb (2003-2010). Here we present results based on data aquired using the Korean 1-m telescope located at Mt.Lemmon, USA. A detailed analysis using a complete set of data, including the one obtained at 4 more telescopes in Ukraine and Slovakia, is in preparation (Andronov et al. 2011).

\section{OBSERVATIONS}

$\mathrm{V}$ and R-band time-series observations were acquired using a $2 \times 2 \mathrm{~K}$ CCD camera mounted on the LOAO $1.0 \mathrm{~m}$ telescope, in Arizona. Given the CCD plate scale of 0.64 arcseconds/pixel at the $\mathrm{f} / 7.5$ Cassegrain focus, the image field-of-view was 22.2 arcminutes square.

Our image data reduction procedure included bias, dark and flat-field calibration, and was performed with the IRAF package CCDRED. Instrumental stellar magnitudes were derived empirically by fitting the pointspread functions (PSFs) of stars using the IRAF package DAOPHOT (Stetson 1987; Massey \& Davis 1992). 


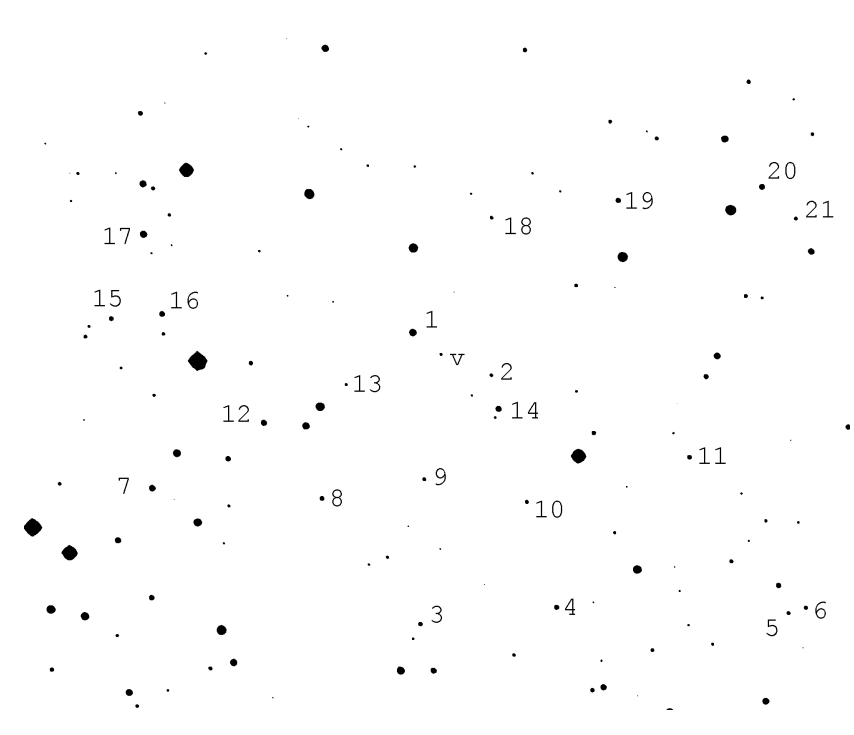

Fig. 1. - Finding chart for 1RXS J180340.0+401214 (marked with "v") and for 21 comparison stars (numbers). North is up and west is left. The size of the field is $7.3^{\prime} \times 4^{\prime}$.

Table 1 shows the journal of observations.

During this campaign, we collected 398 observations (144 measurements in the $\mathrm{V}$ photometric system obtained during 11 nights (21.7 hours) and $254 \mathrm{R}$ data points obtained during 16 nights (38.3 hours)). For 9 nights, we provided two-color photometry as a sequence of measurements with alternating VR filters.

The original observations (HJD, magnitude) are available upon request.

\section{CALIBRATION OF COMPARISON STARS}

In Fig. 1 we present the finding chart for the variable star under study, along with 21 nearby stars used as comparison (sometimes called "reference") objects. According to our notation, the stars " 1 " and "2" coincide with "C1" and "C2" of Gänsicke et al. (2005), who published their approximate values $\mathrm{B}=15^{\mathrm{m}} 1,17^{\mathrm{m}} 9$ and $\mathrm{R}=14^{\mathrm{m}} 1$ and $16^{\mathrm{m}} 7$ for $\mathrm{C} 1$ and $\mathrm{C} 2$, respectively. Henden (2005) presented BVRcIc calibrations for 1077 stars in the field. From his data, the calibration for $\mathrm{C} 1$ is $\mathrm{V}=14.807$ and $\mathrm{Rc}=14.436$ and $\mathrm{V}=17 \mathrm{~m} .432$, and for $\mathrm{C} 2$ is $\mathrm{Rc}=16 \cdot \mathrm{m} 840$. Hereafter, we will use "V" and "R" (or $V_{H}$ and $R_{H}$ ) instead of "V" and "Rc", as a "standard" system, in contrast with the "instrumental" system $V_{i}, R_{i}$.

An accurate calibration is needed for further data reduction. For this purpose, we have chosen 21 stars in the field, the calibration for which was published by Henden (2005). The magnitudes were determined using the "artificial" (mean-weighted) star method (Kim et al. 2004), implemented in the MCV code (Andronov
\& Baklanov 2004). The error of the brightness estimate of the artificial comparison star is 0.0065 in $\mathrm{V}$ and 0.0050 , a value much lower than any other quoted error of the magnitudes of the comparison stars. The smallest magnitude errors are for the "best" comparison star C1 (0. 014 in $\mathrm{V}$ and $0{ }^{\mathrm{m}} 0138$ in R).

Obviously, the variable has a much larger value of the root-mean-squared deviation of the mean (0. 224 in $\mathrm{V}$ and 0.331 in $\mathrm{R}$ ) than any of the comparison stars, because of its intrinsic variability. The typical r.m.s. errors for a single magnitude measurement of the variable star, estimated from the scatter of the comparison stars with similar brightness of $\sim 17^{\mathrm{m}} 5$ is $\sim 0.05$ in $\mathrm{V}$ and $\sim 0.04$ in $\mathrm{R}$, respectively. Because the star is faint, a 1-m telescope was used for the data collection, as smaller ones are not accurate enough.

In Table 2, we list the magnitudes of the comparison stars. The zero-point brightness in the "instrumental' system was defined so that the VR magnitudes of the "main' comparison star C1 were fixed to the values by Henden (2005). Then, using the MCV code (Andronov \& Baklanov 2004), we determine the mean brightness of other comparison stars, as well as the corresponding statistically optimal weights and the scatter estimates. Our results are more accurate than thosee previously published because they are based on 144 and 254 data points instead of one estimate.

We have determined the color transformation coefficients between our "instrumental" ("i") and Henden's (2005) "H" photometric systems. They are listed in Table 3 . The $(V-R)_{H}$ vs. $(V-R)_{i}$ diagram is shown in Fig. 2. A clear trend with a correlation coefficient of $r=0.988 \pm 0.035$ is evident from the plot. This corresponds to a nearly linear dependence, although some points are shifted from the best fit line. However, the slope $b=0.859 \pm 0.030$ differs from unity by $4.6 \sigma$, i.e. it is statistically significant. We also determined the slope $b_{3}=0.868$ of the orthogonal regression (Isobe et al. 1990), which, for such a good correlation, is close to the value of $b$, and thus differs from unity.

After determining the color transformation coefficients, we computed the "corrected instrumental" magnitudes of the variable star and of the comparison stars. They are also listed in Table 2 and they may be used for calibration to the standard photometric systems $\mathrm{V}$, Rc during multi-color observations. However, as only half of the runs are two-color (the rest are either in $\mathrm{V}$, or R), we analyze the two runs in the instrumental system and convert them into the "standard" (V-Rc) color index using the color transformation coefficients listed in Table 3.

Among these comparison stars, we found no variables. However, for other cataclysmic variables from our list, eight new variables were discovered in the apparent vicinities of the main targets: MU Cam (Andronov et al. 2005c), 1RXS J213344.1+510725 (Virnina et al. 2010), TT Ari (Virnina 2010), AK Cnc (Virnina 2011). 
Table 1.

Journal of observations of 1RXS J180340.0+401214: Begining $t_{\text {begin }}$ and ending $t_{\text {end }}$ times, in HJD-2400000, of observations. For the majority of the nights, the observations ended on the next integer JD; the integer part of the starting Julian date JD is used for a legend of the run, e.g., 52752. Number of observations $n$; Magnitude range for individual data points $m_{\max }, m_{\min }$; Nightly mean $\langle m\rangle$ and its accuracy estimate; R.m.s. deviation of the single observation from the mean $\sigma(m) ; \Delta t$ - time resolution.

\begin{tabular}{|c|c|c|c|c|c|c|c|}
\hline$t_{\text {begin }}-t_{\text {end }}$ & $n$ & Duration & range & $\langle m\rangle$ & $\sigma(m)$ & $\Delta t$ & Filter \\
\hline $53667.59175-.64557$ & 26 & 0.054 & $16.923-17.953$ & $17.705 \pm 0.037$ & 0.186 & 185 & $\mathrm{R}$ \\
\hline 53837.81046-.00771 & 28 & 0.197 & $17.462-17.908$ & $17.661 \pm 0.022$ & 0.116 & 245 & $\mathrm{R}$ \\
\hline 53838.84589-.00292 & 21 & 0.157 & $17.404-17.921$ & $17.662 \pm 0.026$ & 0.121 & 645 & $\mathrm{R}$ \\
\hline 53840.91169-.00891 & 14 & 0.097 & $17.359-17.985$ & $17.661 \pm 0.052$ & 0.194 & 645 & $\mathrm{R}$ \\
\hline $53841.79280-.00217$ & 29 & 0.209 & $17.413-18.211$ & $17.742 \pm 0.032$ & 0.172 & 645 & $\mathrm{R}$ \\
\hline $54217.80547-.93847$ & 34 & 0.133 & $17.316-17.899$ & $17.567 \pm 0.024$ & 0.139 & 346 & V \\
\hline 54217.80695-.93617 & 33 & 0.129 & $16.865-17.438$ & $17.159 \pm 0.027$ & 0.153 & 346 & $\mathrm{R}$ \\
\hline $54218.84951-.94651$ & 24 & 0.097 & $17.260-17.806$ & $17.555 \pm 0.034$ & 0.167 & 346 & V \\
\hline $54218.85161-.94861$ & 25 & 0.097 & $16.916-17.297$ & $17.106 \pm 0.025$ & 0.123 & 348 & $\mathrm{R}$ \\
\hline $54258.65449-.79550$ & 36 & 0.141 & $16.623-17.868$ & $17.452 \pm 0.039$ & 0.232 & 346 & V \\
\hline $54258.65646-.79757$ & 36 & 0.141 & $16.743-17.586$ & $17.000 \pm 0.026$ & 0.158 & 339 & $\mathrm{R}$ \\
\hline $54264.66055-.69855$ & 6 & 0.038 & $17.641-17.882$ & $17.791 \pm 0.039$ & 0.095 & 605 & V \\
\hline 54264.67989-.70270 & 4 & 0.023 & $17.146-17.415$ & $17.307 \pm 0.057$ & 0.114 & 656 & $\mathrm{R}$ \\
\hline $54268.71158-.75558$ & 5 & 0.044 & $17.727-17.984$ & $17.845 \pm 0.051$ & 0.114 & 691 & V \\
\hline $54268.71561-.74979$ & 4 & 0.034 & $17.255-17.465$ & $17.369 \pm 0.052$ & 0.104 & 846 & $\mathrm{R}$ \\
\hline 54272.66659-.70559 & 4 & 0.039 & $17.488-17.659$ & $17.562 \pm 0.035$ & 0.071 & 1037 & V \\
\hline $54272.68705-.71151$ & 3 & 0.024 & $17.044-17.238$ & $17.158 \pm 0.058$ & 0.101 & 1056 & $\mathrm{R}$ \\
\hline $54280.65858-.71458$ & 6 & 0.056 & $17.491-18.133$ & $17.803 \pm 0.085$ & 0.208 & 950 & V \\
\hline 54280.67530-.71921 & 5 & 0.044 & $16.999-17.328$ & $17.200 \pm 0.055$ & 0.124 & 948 & $\mathrm{R}$ \\
\hline $54285.66055-.70955$ & 5 & 0.049 & $17.385-17.780$ & $17.557 \pm 0.069$ & 0.153 & 1037 & V \\
\hline $54285.66689-.71547$ & 4 & 0.049 & $17.084-17.173$ & $17.143 \pm 0.021$ & 0.041 & 1049 & $\mathrm{R}$ \\
\hline $54363.77120-.80820$ & 6 & 0.037 & $17.418-17.801$ & $17.572 \pm 0.054$ & 0.132 & 605 & $\mathrm{~V}$ \\
\hline $54363.77521-.80457$ & 5 & 0.029 & $17.008-17.231$ & $17.137 \pm 0.039$ & 0.086 & 632 & $\mathrm{R}$ \\
\hline $54402.56789-.68488$ & 8 & 0.117 & $17.426-17.941$ & $17.658 \pm 0.072$ & 0.203 & 643 & $\mathrm{R}$ \\
\hline $54402.57158-.68157$ & 8 & 0.109 & $17.670-18.354$ & $17.984 \pm 0.079$ & 0.223 & 605 & $\mathrm{~V}$ \\
\hline $54405.56547-.64347$ & 10 & 0.078 & $17.922-18.277$ & $18.120 \pm 0.039$ & 0.122 & 605 & V \\
\hline $54405.56983-.64704$ & 9 & 0.077 & $17.410-17.870$ & $17.619 \pm 0.051$ & 0.154 & 677 & $\mathrm{R}$ \\
\hline
\end{tabular}

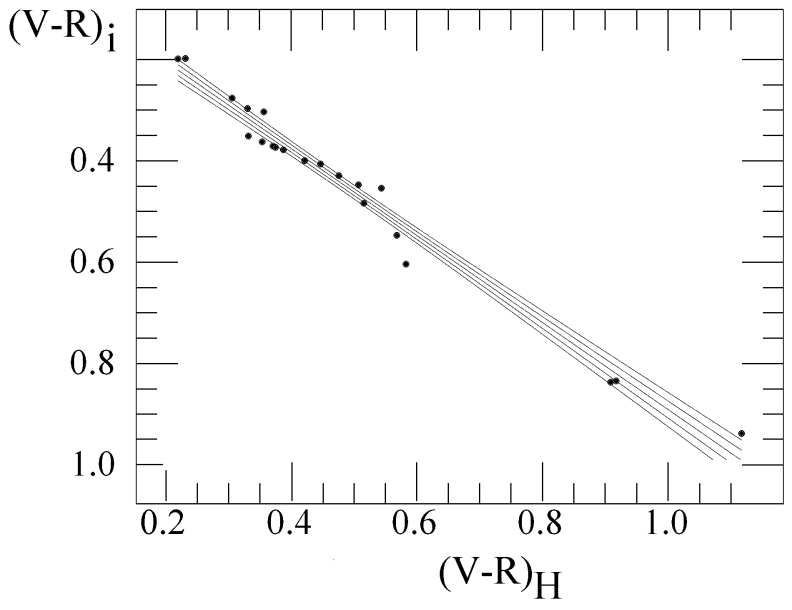

Fig. 2.- The $(V-R)_{H}$ vs. $(V-R)_{i}$ diagram, the corresponding linear fit and the $\pm 1 \sigma$ and $\pm 2 \sigma$ scatter lines.

\section{LONG-TERM LUMINOSITY CHANGES}

The time light curve of RXJ 1803 is shown in Fig. 3 for the observations in $\mathrm{V}$ and $\mathrm{R}$. The scatter is mainly due to orbital and spin variability, as the accuracy estimate of a single point is $\sim 0.04$. One may note that the mean level for the observations undergoes switches between two values, which one could mark as "high" and "low" luminosity states, similar to other intermediate polars. For our observations, the "high" state occurs for the nights 2454217-363 and lasted at least 245 days. This is a lower limit, as the transitions between the luminosity states were not observed. The sample mean values of brightness are $\bar{V}_{i}=17.564 \pm 0 .{ }^{\mathrm{m}} 018$, $\bar{R}_{i}=17^{\mathrm{m}} \cdot 112 \pm 0 .{ }^{\mathrm{m}} 015$ for 126 and 119 data points, respectively. The corresponding color index is $(V-R)_{i}=$ $0 \mathrm{~m} 452 \pm 0 . \mathrm{m} 023$. For the two last runs in a "low" state, the mean brightness values are $\bar{V}_{i}=18{ }^{\mathrm{m}} 060 \pm 0 .{ }^{\mathrm{m}} 043$, $\bar{R}_{i}=17^{\mathrm{m}} 637 \pm 0 \cdot{ }^{\mathrm{m}} 042,(V-R)_{i}=0 \cdot{ }^{\mathrm{m}} 422 \pm 0.060$. Although the system appears to be slighly "redder" in the high state, like a magnetic cataclysmic variable (asynchronous polar) BY Cam (Andronov et al. 2008) and as opposed to "non-magnetic" cataclysmic variables, 
Table 2.

Calibration of the comparison stars. The index "H" corresponds to the Henden's (2005) estimates, "i" to our "instrumental" system, and "ci" to the "corrected instrumental" systems.

\begin{tabular}{cccccccccc}
\hline Star & $V_{H}$ & $R_{H}$ & $(V-R)_{H}$ & $V_{i}$ & $R_{i}$ & $(V-R)_{i}$ & $V_{c i}$ & $R_{c i}$ & $(V-R)_{c i}$ \\
\hline RXJ & 17.880 & 17.288 & 0.592 & $17.591 \pm 0.022$ & $17.415 \pm 0.022$ & 0.176 & 17.579 & 17.404 & 0.175 \\
C1 & 14.807 & 14.436 & 0.371 & $14.807 \pm 0.001$ & $14.436 \pm 0.001$ & 0.371 & 14.793 & 14.396 & 0.397 \\
C2 & 17.423 & 16.840 & 0.583 & $17.459 \pm 0.007$ & $16.856 \pm 0.002$ & 0.604 & 17.442 & 16.781 & 0.662 \\
C3 & 16.632 & 16.156 & 0.476 & $16.646 \pm 0.004$ & $16.217 \pm 0.002$ & 0.429 & 16.632 & 16.168 & 0.463 \\
C4 & 16.194 & 15.863 & 0.331 & $16.184 \pm 0.004$ & $15.888 \pm 0.002$ & 0.297 & 16.171 & 15.858 & 0.313 \\
C5 & 16.975 & 16.468 & 0.507 & $17.033 \pm 0.004$ & $16.586 \pm 0.002$ & 0.447 & 17.018 & 16.535 & 0.483 \\
C6 & 17.044 & 16.500 & 0.544 & $17.059 \pm 0.008$ & $16.605 \pm 0.002$ & 0.454 & 17.043 & 16.552 & 0.491 \\
C7 & 15.353 & 14.996 & 0.357 & $15.359 \pm 0.001$ & $15.056 \pm 0.001$ & 0.303 & 15.346 & 15.026 & 0.320 \\
C8 & 16.373 & 16.141 & 0.232 & $16.321 \pm 0.008$ & $16.123 \pm 0.003$ & 0.198 & 16.308 & 16.108 & 0.200 \\
C9 & 17.056 & 16.634 & 0.422 & $17.054 \pm 0.005$ & $16.653 \pm 0.002$ & 0.400 & 17.039 & 16.608 & 0.431 \\
C10 & 17.518 & 16.601 & 0.917 & $17.507 \pm 0.008$ & $16.672 \pm 0.002$ & 0.834 & 17.487 & 16.563 & 0.924 \\
C11 & 16.719 & 16.151 & 0.568 & $16.791 \pm 0.003$ & $16.244 \pm 0.002$ & 0.547 & 16.775 & 16.177 & 0.598 \\
C12 & 15.846 & 15.330 & 0.516 & $15.852 \pm 0.003$ & $15.368 \pm 0.001$ & 0.484 & 15.837 & 15.311 & 0.526 \\
C13 & 18.159 & 17.042 & 1.117 & $18.105 \pm 0.011$ & $17.167 \pm 0.003$ & 0.938 & 18.085 & 17.042 & 1.042 \\
C14 & 15.499 & 15.279 & 0.220 & $15.442 \pm 0.004$ & $15.243 \pm 0.002$ & 0.199 & 15.429 & 15.228 & 0.201 \\
C15 & 17.044 & 16.135 & 0.909 & $17.099 \pm 0.007$ & $16.262 \pm 0.001$ & 0.837 & 17.080 & 16.153 & 0.927 \\
C16 & 15.844 & 15.538 & 0.306 & $15.870 \pm 0.003$ & $15.594 \pm 0.001$ & 0.276 & 15.857 & 15.567 & 0.289 \\
C17 & 14.903 & 14.456 & 0.447 & $14.939 \pm 0.003$ & $14.533 \pm 0.001$ & 0.406 & 14.924 & 14.487 & 0.437 \\
C18 & 17.083 & 16.729 & 0.354 & $17.091 \pm 0.005$ & $16.728 \pm 0.002$ & 0.363 & 17.077 & 16.688 & 0.388 \\
C19 & 16.210 & 15.835 & 0.375 & $16.247 \pm 0.004$ & $15.874 \pm 0.002$ & 0.373 & 16.233 & 15.833 & 0.399 \\
C20 & 15.760 & 15.428 & 0.332 & $15.837 \pm 0.002$ & $15.486 \pm 0.002$ & 0.351 & 15.823 & 15.448 & 0.374 \\
C21 & 17.034 & 16.646 & 0.388 & $17.093 \pm 0.004$ & $16.715 \pm 0.002$ & 0.378 & 17.079 & 16.674 & 0.405 \\
\hline
\end{tabular}

Table 3.

Characteristics of the color transformation coefficients between the standard "H" (Henden 2005) and our instrumental "i" systems. The linear fit is taken in a form $y=a+b \cdot x=\bar{y}+b \cdot(x-\bar{x})$, where $\bar{x}$ is a sample mean of $x$. The last form corresponds to the best accuracy of the "zero point" $\bar{y}$ as compared to $a$. The number of comparison stars used is $n=21$. Also listed are the slope of the line of the orthogonal regression $b_{3}$, the "unit weight error" $\sigma_{0}$, the correlation coefficient $r$ and the parameter $r / \sigma_{r}=b / \sigma_{b}$.

\begin{tabular}{ccrrrrrrrr}
\hline$x$ & $y$ & \multicolumn{1}{c}{$a$} & \multicolumn{1}{c}{$b$} & \multicolumn{1}{c}{$b_{3}$} & \multicolumn{1}{c}{$\bar{x}$} & \multicolumn{1}{c}{$\bar{c} \sigma_{0}$} & \multicolumn{1}{c}{$r$} & $r / \sigma_{r}$ \\
\hline$(V-R)_{H}$ & $(V-R)_{i}$ & 0.032 & 0.859 & 0.868 & 0.489 & 0.452 & 0.031 & 0.988 & 28.4 \\
& & \pm 0.016 & \pm 0.030 & & \pm 0.008 & \pm 0.007 & & \pm 0.035 & \\
$(V-R)_{H}$ & $V_{i}-V_{H}$ & 0.017 & -0.004 & -0.004 & 0.489 & 0.015 & 0.040 & -0.026 & -0.1 \\
& & \pm 0.021 & \pm 0.039 & & \pm 0.051 & \pm 0.009 & & \pm 0.229 & \\
$(V-R)_{H}$ & $R_{i}-R_{H}$ & -0.014 & 0.136 & 0.139 & 0.489 & 0.052 & 0.035 & 0.682 & 4.1 \\
& & \pm 0.018 & \pm 0.036 & & \pm 0.051 & \pm 0.010 & & \pm 0.168 & \\
$(V-R)_{i}$ & $(V-R)_{H}$ & -0.025 & 1.137 & 1.153 & 0.452 & 0.489 & 0.036 & 0.988 & 28.4 \\
& & \pm 0.020 & \pm 0.040 & & \pm 0.044 & \pm 0.009 & & \pm 0.035 & \\
$(V-R)_{i}$ & $V_{H}-V_{i}$ & -0.010 & -0.011 & -0.012 & 0.452 & -0.015 & 0.040 & -0.058 & -0.3 \\
& & \pm 0.022 & \pm 0.044 & & \pm 0.044 & \pm 0.008 & & \pm 0.229 & \\
$(V-R)_{i}$ & $R_{H}-R_{i}$ & 0.014 & -0.148 & -0.153 & 0.452 & -0.052 & 0.036 & -0.644 & -3.7 \\
& & \pm 0.020 & \pm 0.040 & & \pm 0.044 & \pm 0.010 & & \pm 0.175 & \\
\hline
\end{tabular}

this difference may not be statistically significant because only two short runs were obtained in the "low" state with 18 and 17 data points in $V_{i}$ and $R_{i}$, respectively. The magnitude difference between the "high" and "low" states is $\Delta V=0 \mathrm{~m} 50$. The color index in the "high" luminosity state reduced to the standard photometric system is $(V-R)_{H}=0.489$. This value is close to that of 0.452 in the instrumental system.

\section{ORBITAL AND SPIN VARIABILITY}

In the discovery paper, Gänsicke et al. (2005) noted an orbital period of RXJ 1803 of 160.2101 minutes $=0.111257$ days based on spectral observations, 


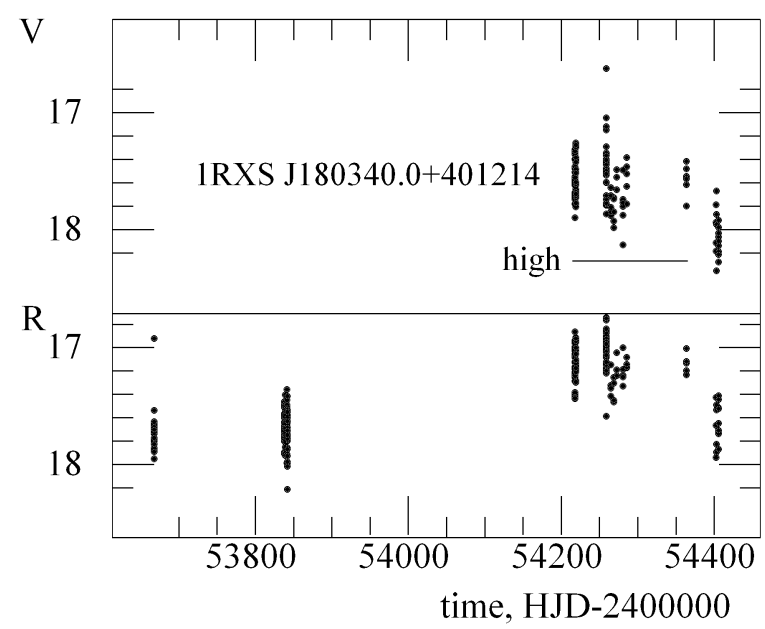

Fig. 3. - The light curves in the instrumental VR photometric systems. The horizontal line denotes an interval of observations, when the system was in its high luminosity state.

which is not well pronounced in the photometric data. The sine fit for the data at the "high" state corresponds to a "semi-amplitude" in $V_{i}$ of $r=0.053 \pm 0.026$, i.e., only $2 \sigma$. This is not statistically significant, so the orbital variability may be neglected. In $R_{i}, r=$ $0{ }^{\mathrm{m}} 005 \pm 0.021$ is even conciderably smaller.

The published values of the spin period are 1520.510 $\mathrm{sec}=0.01759850$ days (frequency $f=1 / P=56.82304$ cycles/day) (Gänsicke et al. 2005) and 0.01764120(3) days $(f=56.68549$ cycles/day) (Teichgraeber et al. 2007). This apparently small difference causes a cycle count error by unity every 7.3 days, or 50 cycles per year, thus the determination of spin periods needs improvement.

In Fig. 4 we show a periodogram $S(f)$ in the range 45-65 cycles/day, in the vicinities of the expected peak, for the $R_{i}$ data. The test function $S(f)$ is the square of the correlation coefficient between the observations and the values calculated using the sine fit with a trial frequency (see Andronov 1994, 2003) for details). The highest peak corresponds to a frequency of $f=55.8201 \pm 0.0008$ cycles/day, spin period $P_{\text {spin }}=0.01791471 \pm 0.00000025$ semi-amplitude $r=0^{\mathrm{m}} \cdot 110 \pm 0^{\mathrm{m}} 019$ and initial epoch for the maximum $T_{0}=$ HJD $2454246.16667 \pm 0.00049$. This frequency is a daily bias of the values previously published by Gänsicke et al. (2005) and Teichgraeber et al. (2007). In the range of frequencies 56-57 cycles/day, the highest peak corresponds to the value of $f=56.8250 \pm$ 0.0008 cycles $/$ day, $P_{\text {spin }}=0.01759790 \pm 0.00000025$ (i.e., close to the value published by Gänsicke et al. 2005, but not by Teichgraeber et al. 2007), $r=$ $0 .{ }^{\mathrm{m}} 105 \pm 0 .{ }^{\mathrm{m}} 020, T_{0}=$ HJD $2454246.15985 \pm 0.00051$. For the $V_{i}$ data, the amplitude is significantly smaller and its estimate is equal to $r=0.055 \pm 0.025$, $\left(T_{0}=\right.$ HJD 2454248.35148 \pm 0.00131$)$ (our period) or to $r=0 .{ }^{\mathrm{m}} 063 \pm 0 .{ }^{\mathrm{m}} 025,\left(T_{0}=\right.$ HJD $\left.2454248.35871 \pm 0.00113\right)$ (period of Gänsicke et al. 2005). Thus from the "high" state data, even with two colours, we cannot choose the proper cycle counting.

For the "low" state at the beginning of the campaign, only one-color $R_{i}$ data are available. The periodogram for 4 runs nearly subsequent in date (JD 2453837-53841), containing 92 data points, has a significant highest peak at $f=56.806 \pm 0.018$ cycles $/$ day, $P_{\text {spin }}=0$ d.0176038 \pm 0 d $0000055, r=$ $0 .{ }^{\mathrm{m}} 105 \pm 0.019, T_{0}=$ HJD $2453839.86478 \pm 0.00053$. For the first run, separated from the next season, $T_{0}=$ HJD $2453667.62112 \pm 0.00092$ with a smaller amplitude $r=0{ }^{\mathrm{m}} 075 \pm 0.024$.

\section{IMPROVED SPIN PERIOD}

Using the published maxima timing values (6 of Gänsicke et al. 2005; one of Teichgraeber et al. 2007) and four mean maxima determined with the current work $(2453667.62112,2453839.86478,2454246.15985$, 2454248.35871), we have tried to determine a corrected value of the spin period. However, we disclosed unexpected findings: the maxima timings of Gänsicke et al. (2005) do not fit their period of 0 d.01759850 mentioned above. If taking the first minimum as an initial epoch (unfortunately, no epoch was provided in their paper), the phases of 5 other maxima are very close $(\sim 0.56)$. To analyze the $\mathrm{O}-\mathrm{C}$ diagram, we have used the PerMin software (Andronov 1991), which computes the periodogram for the moments of "characteristic events" (for intermediate polars, photometric maxima). We show this periodogram in Fig. 5. Separately, we have computed similar periodograms for all the 11 timings and for last 5 (4 ours and one of of Teichgraeber et al. 2007). The best fit periods and the initial epochs are $P=0 \mathrm{~d} 017600489(41)$, $T_{0}=2452854.61979(8)$ for the observations of Gänsicke et al. (2005) and $P=0$ d $017599193(23)$ and $T_{0}=$ 2453977.7540(3). The last digit under brackets is an error estimate. This difference is statistically significant $(28 \sigma)$ and does not allow to constrain a joint ephemeris for all the 11 timings. Assuming that this difference is due to period variations, which are sometimes seen in the intermediate polars (cf., Andronov et al. 2005ab; Kim et al. 2005b), we estimate the period derivative $\dot{P} \approx\left(P_{2}-P_{1}\right) /\left(T_{2}-T_{1}\right)=-1.15(4) \cdot 10^{-9}$. However, the corresponding quadratic term in the ephemeris does not allow to make a good fit for all the maxima. So we need intensive monitoring surveys to properly address the question of period variations.

\section{CONCLUSIONS}

The main results of our study of RXJ 1803 can be summarized as follows: 


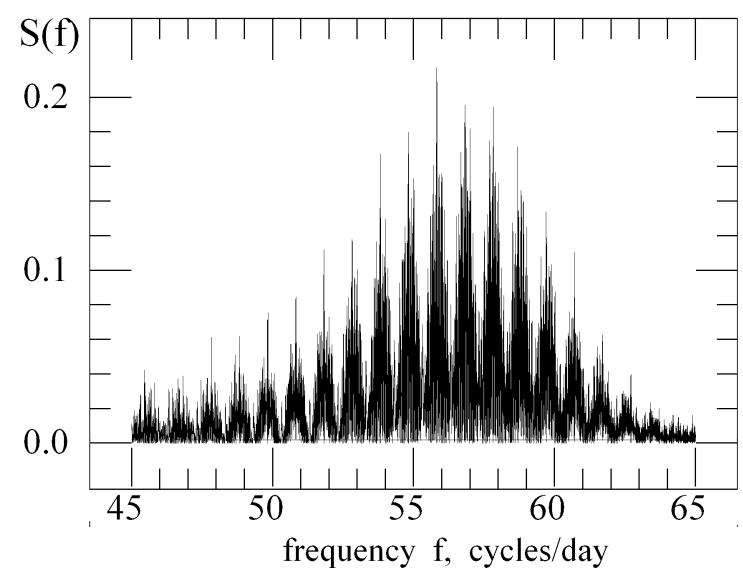

Fig. 4. - The periodograms for the $R_{i}$ observations at the "high" state.

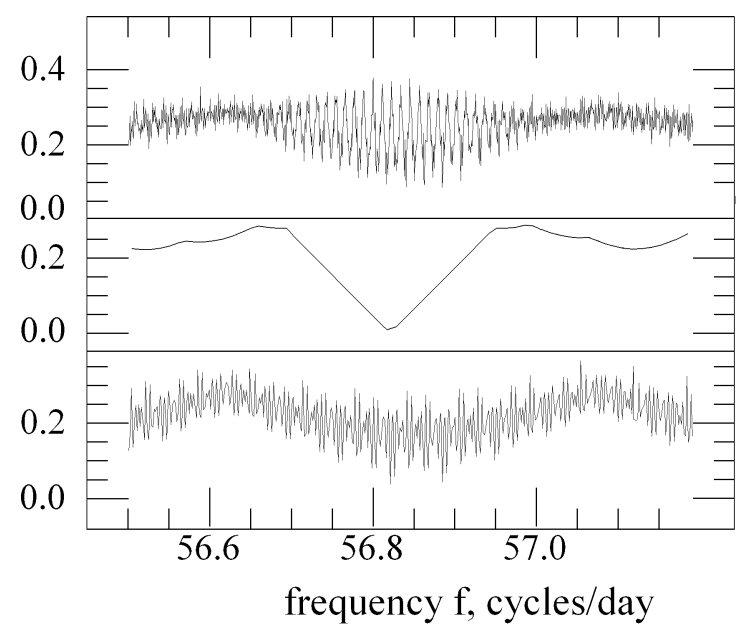

Fig. 5. - The periodograms for the spin maxima moments: all 11 (up), first 6 of Gänsicke et al. (2005) (middle) and last 5 (bottom).

- We discover "high" and "low" states;

- We found absence of statistically significant variability of the color index with changes in luminosity;

- We argued that orbital variability is not statistically significant;

- We determined the maxima timings which allowed us to compute the ephemeris for the time interval of our observations;

- We detected spin period variations. However, the character of this variability caused by rotational evolution of the magnetic white dwarf in the system needs further observations;
- We computed the color transformation coefficients for our photometric systems, as well as improved secondary photometric standards.

\section{ACKNOWLEDGMENTS}

This work was supported by the Chungbuk National University Grant in 2008 and partially supported by the Ministry of Education and Science of Ukraine. We thank the Korean Astronomy Observatory to support the observation with Mt. Lemmon. YK acknowledges the support of Chungbuk National University, which enabled him to contribute to this paper while a visiting professor at the Department of Physics and Astronomy, University of California, Irvine. He wishes to express his appreciation for the hospitality of the staff of the department.

\section{REFERENCES}

Andronov, I. L. 1991, Determination of the Period of a Variable Signal from the Times of Characteristic Events, Kinem. Phys. Celest. Bodies, 7, 70

Andronov, I. L. 1994, (Multi-)Frequency Variations of Stars. Some Methods and Results, Odessa Astron. Publ., 7, 49

Andronov, I. L. 2003, Multiperiodic versus Noise Variations: Mathematical Methods, ASPC, 292, 391

Andronov, I. L. 2007, Structure and Evolution of Magnetic Cataclysmic Variables, ASPC, 362, 200

Andronov, I. L. 2008, Gravi-Magnetic Rotators in Cataclysmic Binary Stars, JPhSt, 12, 2902

Andronov, I. L., Antoniuk, K. A., Augusto, P., Baklanov, A. V., Chinarova, L. L., Chochol, D., Efimov, Yu. S., Gazeas, K., Halevin, A. V., Kim, Y., Kolesnikov, S. V., Kudashkina, L. S., Marsakova, V. I., Mason, P. A., Niarchos, P. G., Nogami, D., Ostrova, N. I., Patkos, L., Pavlenko, E. P., Shakhovskoy, N. M., Tremko, J., Yushchenko, A. V., \& Zola, S. 2003, Inter-Longitude Astronomy Project: Some Results and Perspectives, A\&AT, 22, 793

Andronov, I. L., Antoniuk, K. A., Baklanov, A. V., Breus, V. V., Burwitz, V., Chinarova, L. L., Chochol, D., Dubovsky, P. A., Han, W., Hegedus, T., Hric, L., Kim, C.-H., Kim, Y., Kolesnikov, S. V., Kudzej, I., Liakos, A., Niarchos, P. G., Oksanen, A., Patkos, L., Petrik, K., Pit', N. V., Shakhovskoy, N. M., Virnina, N. A., Yoon, J., \& Zola, S. 2010, "Inter-Longitude Astronomy" (ILA) Project: Current Highlights And Perspectives. I. Magnetic vs. Non-Magnetic Interacting Binary Stars, Odessa Astron. Publ., 23, 8

Andronov, I. L., Antoniuk, K. A., Breus, V. V., Chinarova, L. L., Han, W., Jeon, Y. B., Kim, Y.-G., Kolesnikov, S. V., Oh, J. Y., Pavlenko, E. P., \& Shakhovskoy, N. M. 2008a, Idling Magnetic White 
Dwarf in the Synchronizing Polar BY Cam. The Noah-2 Project, CEJPh, 6, 385

Andronov, I. L., \& Baklanov, A. V. 2004, Astron. School's Report, 5, 264; http://uavso.pochta.ru/mcv

Andronov, I. L., Chinarova, L. L., Han, W., Kim, Y., \& Yoon, J.-N. 2008b, Multiple Timescales in Cataclysmic Binaries. The Low-Field Magnetic Dwarf Nova DO Draconis, A\&A, 486, 855

Andronov, I. L., Kim, Y. G., Shin, J.-H., \& Jeon, Y. B. 2005a, O-C Analysis of the Intermediate Polar BG CMi, ASPC, 335, 355

Andronov, I. L., Ostrova, N. I., \& Burwitz, V. 2005b, O-C Analysis of FO Aqr, "King of Intermediate Polars", ASPC, 335, 229

Andronov, I. L., Ostrova, N. I., Kim, Y.-G., \& Burwitz, V. 2005c, Two-Color VR CCD Photometry of Old Nova V603 Aquilae, JASS, 22, 211

Anzolin, G.,deMartino, D., Bonnet-Bidaud, J.-M., Mouchet, M., Gansicke, B. T., Matt, G., \& Mukai, K. 2008, Two New Intermediate Polars with a Soft X-Ray Component, A\&A, 489, 1243

Gansicke, B. T., Marsh, T. R., Edge, A., RodriguezGil, P., Steeghs, D., Araujo-Betancor, S., Harlaftis, E., Giannakis, O., Pyrzas, S., Morales-Rueda, L., \& Aungwerojwit, A. 2005, Cataclysmic Variables from a ROSAT/2MASS Selection I. Four New Intermediate Polars, MNRAS, 361, 141

Hellier, C. 2001, Cataclysmic Variable Stars. How and Why They Vary, Springer Berlin

Henden, A. 2005, ftp://ftp.aavso.org/public/calib /j1803.dat

Isobe, T., Feigelson, E. D., Akritas, M. G., \& Babu, G. J. 1990, Linear Regression in Astronomy, ApJ, 364, 104

Kim, Y., Andronov, I. L., Cha, S. M., Chinarova, L. L., \& Yoon, J. N. 2009, Nova-Like Cataclysmic Variable TT Arietis. QPO Behaviour Coming Back from Positive Superhumps, A\&A, 496, 765

Kim, Y., Andronov, I. L., \& Jeon, Y.-B. 2004, CCD Photometry Using Multiple Comparison Stars, JASS, 21, 191

Kim, Y.-G., Andronov, I. L., Park, S.-S., Chinarova, L. L., Baklanov, A. V., \& Jeon, Y.-B. 2005a, TwoColor VR CCD Photometry of the Intermediate Polar 1RXS J062518.2+733433, JASS, 22, 197

Kim, Y.-G., Andronov, I. L., Park, S. S., \& Jeon, Y.-B. 2005b, Orbital and Spin Variability of the Intermediate Polar BG CMi, A\&A, 441, 663

Massey, P., \& Davis, L. E. 1992, A User's Guide to Stellar CCD photometry with IRAF

Norton, A. J., Beardmore A. P., Allan, A., \& Hellier, C. 1999, YY Draconis and V709 Cassiopeiae: Two Intermediate Polars with Weak Magnetic Fields, A\&A, 347, 203
Patterson, J. 1994, The DQ Herculis stars, PASP, 106, 209

Ritter, H., \& Kolb U. 2003, Catalogue of Cataclysmic Binaries, Low-Mass X-Ray Binaries and Related Objects, A\&A, 404, 301; living Edition: http://physics.open.ac.uk/RKcat/

Samus, N. N., et al. 2009, General Catalog of Variable Stars, yCat, 102025S;

http://www.sai.msu.su/groups/cluster/gcvs/

SIMBAD, 2011, http://simbad.u-strasbg.fr

Stetson, P. B. 1987, DAOPHOT - A Computer Program for Crowded-Field Stellar Photometry, PASP, 99,191

Teichgraeber,C., Wood,M.A., Patterson,J., Monard,B., Rea,R., \& Kemp,J. 2007, Updates on Two New Cataclysmic Variable Systems: 1RXS J173021.5-055933 and 1RXS J180340.0+401214, JSARA, 1, 24

Virnina, N. A. 2010, Three New Variable Stars near TT Ari, Open European Journal on Variable stars, 124,1

Virnina, N. A. 2011, Two New EW-type Eclipsing Variables Near AK Cnc, Open European Journal on Variable stars, 132, 1

Virnina, N, A. Andronov, I. L., Dubovsky, P. A., Kudzej, I. 2010, Two New Eclipsing Variable Stars in the Field of RX J2133,Open European Journal on Variable stars, 119, 1

Warner, B. 1995, Cataclysmic Variable Stars, Cambridge Univ. Press

Watson, C. L. 2006-2011, AAVSO International Variable Star Index VSX, The Journal of the American Association of Variable Star Observers, 35, 318 